\title{
Distributed and secure cooperative engineering in virtual plant production
}

\author{
Jan Woerner and Heinz Woern \\ Universitaet Karlsruhe(TU), Department of Computer Science, Institute for Process Control \\ and Robotics (IPR), 76128 Karlsruhe, Germany.Email: woerner@ira.uka.de
}

\begin{abstract}
Today, cooperative engineering in the fields of plant production is a time consuming and expensive issue. The integration process of product data and models into the planning and engineering of the production facilities and vice versa is a complex task. Furthermore, these integration processes are repeated in an iterative manner at every level or phase of the plant production project in a supply network of suppliers, turnkey suppliers and manufacturers. The diversity of planning, design and simulation tools, as well as their varying model structures result in completely different interfaces. The globally located cooperation partners and the highly distributed sub-models throughout the supply network cannot be integrated into one complete model. As a result, a consistent digital representation of the complete production facility is not available, nowadays. Against this background, a new web service based platform providing developed methods for cooperative plant production within virtual engineering is presented in this paper. It supports the protection of knowledge as well as efficient role and project dependent encrypted data exchange with a definable level of security based on XML. An integration into an existing legacy software architecture is exemplified with popular engineering software.
\end{abstract}

Keywords

Cooperation, Data Security, Virtual Engineering, Digital Factory, Web Services.

\section{INTRODUCTION}

The trend of the plant production market leads to the development of digital factories, where the planning, simulation and engineering of plant subsystems is globally performed in the virtual world. Therefore, a complete and consistent model of a plant is required. Numerous modelling, planning and simulation tools are used to create these partial models, today. In the most cases a complete model of a whole production facility is not available. The diversity of planning, design and simulation tools, as well as their varying model structures result in completely different interfaces. For this reason, an integration of all the distributed globally located submodels is very complex. In addition, data related to existing plants, is often either not available or not complete. 
As a consequence, a decentralised system based on a service oriented architecture is developed, supporting an integration of all suppliers into all different phases within the plant production process. This distributed system thus enables an improvement of cooperative plant production and the plant itself, addressed by [1].

The infrastructure for vendor integrated decentralised cooperative work (IVM) described in [1] contains model managing and integration with clearly definable roles and project dependent views to models and events for different manufacturers and suppliers. Encryption and signing methods integrated into a web service structure enable a secure, reliable and parallel, transparent transmission of data that is required for efficient methods in cooperative work. The support of asynchronous and synchronous data transfer enables the flexibility required for cooperative work.

On this account, this paper describes based on concerning state of the art results the research regarding modern cooperation in plant production processes. Afterwards the used technologies like secure web services are introduced, based on a service-oriented architecture (SOA).

To demonstrate the usability, the cooperative work supporting and enhancing software is presented in an example test case within a plant production environment. Finally, conclusions on this research and developments and plans to extend it are considered.

\section{STATE OF THE ART}

The value of cooperative engineering enabling software tools to communicate in the fields of manufacturing is recognised and solutions are partly available. However these solutions do not meet the requirements for cooperative work in the field of plant production processes. Joseph Bianco and Jay P. Harrison point out in [2], "The ability to perform concurrent engineering activities in widely separated IPPD (Integrated Product and Process Design) teams becomes increasingly important." An example for a proprietary distributed simulation system is given, implying a common hard - and software. It intents the exchange of the existing hardware and software in both cooperative partners companies. This will never be enforceable.

Barry [3] addresses integration among so called manufacturing execution systems (MES) and enterprise information systems (EIS) or enterprise information portals (EIP). These do not consider the properties and the requirements of distributed location of growing complex models in special. On the one hand, the approach of Joseph Bianco and Jay P. Harrison [2] supports these properties and requirements, but takes as well as Smirnov in [4] not a connectivity via an existing and naturally insecure network, like the Internet, into account. On the other hand engineering, e.g. planning or simulation, in the virtual world is not possible without exchanging at least views of the production facility models.

The exchange and interaction of simulation object models is supported by the high level architecture interface (HLA) for simulation applications, described in [5]. HLA does not well support cooperative work, because on the one hand there is no knowledge protection included and on the other hand from a huge pool of engineering applications only simulation applications are considered. Its general purpose is simulation reuse and interoperability. The HLA was developed under the 
leadership of the Defence, Modelling and Simulation Office (DMSO) to support reuse and interoperability across the large numbers of different types of simulations that are developed and have to be maintained. It mainly requires every partner willing to publish all of the needed models. Such a way of model exchange is often prohibited by the companies' security and knowledge protection policies [6]. The importance of trust between the partners of a virtual cooperation consortium is described by Salmi in [7] within an explanation of a cooperative ordering system. The difference between this ordering system and the cooperative work supporting system described in this paper is the complexity of the sequential changing models in plant production demanding a different consideration and methodology.

For that reason, the HLA-interface is possible to use within the fields of connecting local area simulation applications. However, for cooperative engineering purposes additional methods, which meet the requirements of the manufacturer of the product as well, as the turnkey or the sub-suppliers, are needed.

In $[8,9] \mathrm{P}$. Leitão gives an overview of these needs for cooperative distributed manufacturing and proposals for possible inter-company cooperation mechanisms including XML-based communication. $\mathbb{P}$. Leitão uses in [8] a CORBA infrastructure. The main problems existing with CORBA concerning inter-company cooperative work are security related. As a matter of fact firewalls intercept the IIOP protocol based messages and the ORB cores are blocked. ORB vendors try to overcome this drawback by introducing XML based protocols for inter ORB communication, but no standard is available.

Further arguments for a service-oriented architecture based on web services being used in a cooperative work enhancing software system is the open source availability and the extensibility to enable encrypted data transfer. At the moment of writing the paper in hand, only a few web services exist in the field of information technologies. No web service based application for support of plant production engineering has shown up, yet. Additionally only a few small demonstration applications exist, partly implementing the security issues described in this paper. No web service application exist, including all relevant security methods to enable a reliable communication. Against this background the cooperation properties in plant production and appropriate communication platforms are investigated in more detail.

\section{COOPERATIVE PLANT PRODUCTION}

Cooperative work in plant production projects requires an expensive integration of product data into the development environment of the concerning plant, today. The expensive integration is caused by the use of paper ware, incompatible software data formats, different semantics and/or syntax. Agile reactions on changes are expensive or even impossible because of long term processes.

The important early integration of suppliers and sub-suppliers is hardly taken into account within concurrent engineering. The result is a time and money consuming multiple iteration process in each phase. In the most cases time delays are the result. Therefore even phase spanning processes are delayed or iterations occur. This includes the exchange of planning and engineering data from all 
suppliers and sub-suppliers involved in the process throughout all phases in the fields of plant production.

An identification of the plant production phases, shown in Figure 1 was consolidated with a developed role description, including the main tasks, the location and the used software into a role and phase model for plant production.

Based on the developed role and phase model explained in detail in [6], abstractly shown in Figure 2, a concept for cooperative plant production was developed. In this more general scenario several manufacturers cooperate with several technology suppliers, that can also work as sub suppliers divided into line builders or turn key suppliers and engineering houses. These can also be subsuppliers or turn key suppliers dependent on the project.

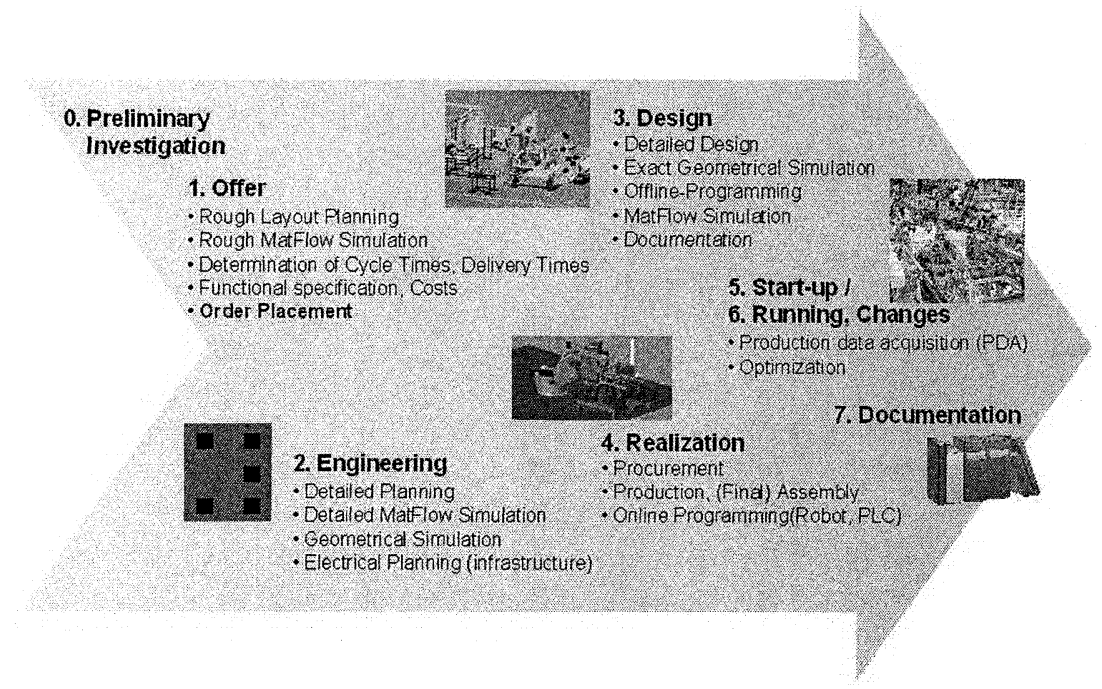

Figure 1 - Phases in plant production

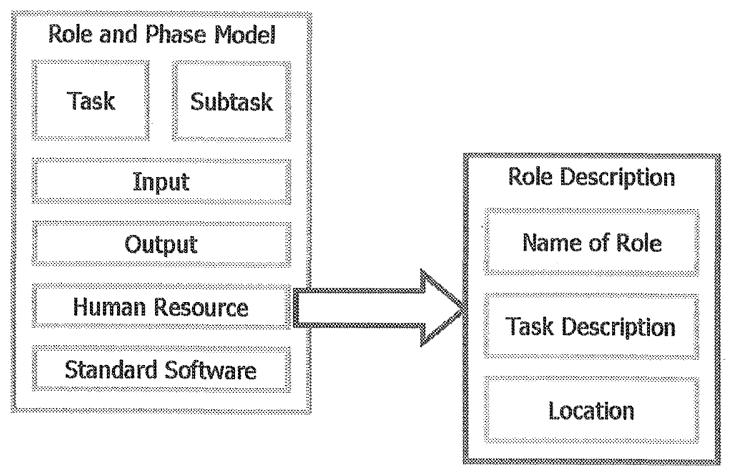

Figure 2 - Role and phase model [6] 
Obviously, many different and complex scenarios, as shown in Figure 3, for cooperation projects exist, but all scenarios have the same requirements regarding the exchange of models: On the one hand the partners should be enabled to work on those parts of the models they are urged to do, on the other hand each company wants to protect important knowledge $[3,7]$. Therefore the companies prerequisite a functionality facilitating a secure, encrypted model exchange with their existing software system, considering the above described requirements.

Against this background a precondition for establishing cooperative engineering is a distributed system, working on globally located models. In addition a published description of the model class is required to enable the integration process by developing interfaces to the company in-house software systems. One solution for deriving such a common model class description is making use of XML. Different $\mathrm{XML}$-based models can be processed independently from the originating software.

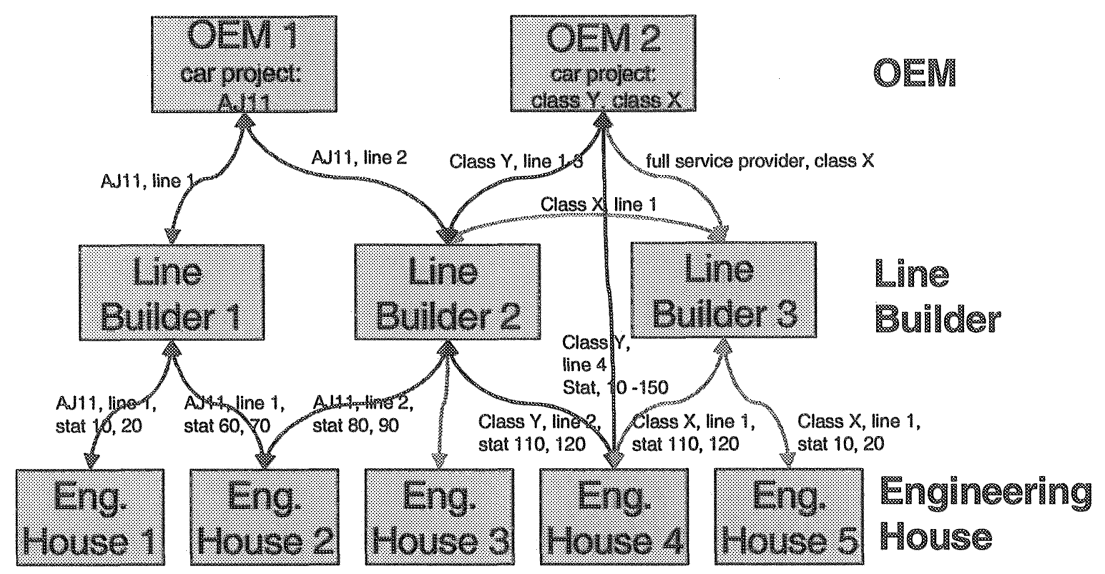

Figure 3 Co-operation hierarchy in plant production [1]

For these types of XML-based models a view enabling mechanism was developed. This view mechanism reduces the models and data to the really required volume. With this mechanism role and project dependent views on the models can be dynamically built on available models. An appropriate user interface enables an easy administration of the views. These model views enable knowledge protection as well as a non redundant data exchange that is required for cooperative engineering. Not necessary parts of the models are not exchanged or published.

After user authentication and authorisation because of a certain role in the respective project the requested model is optimised and filtered by the previously administrated model related view mechanism.

The creation of a view to a model requires a model (re -) integration process described in $[10,11]$. This process enables each user to (re -) integrate previously received sub-models from cooperation project partners. For this model integration process a model management is needed providing model life cycle control such as versioning etc. explained in detail within $[10,11]$.

To handle the increasing complexity of models and objects in plant production an easy scalable and modular system is needed. In such a distributed problem- 
solving environment the tasks are divided in a number of modules or units. This makes persuasive software engineering faster and more agile. The property of platform spanning implementation makes adoptions and extensions easier. The cooperative engineering software has to provide an infrastructure technology enabling an integration of heterogeneous applications and legacy architectures already existing in the companies.

Existing popular standard technologies enable a global, world wide cooperative work environment. Industrial driven surveys finally resulted in the application of a highly distributed approach.

\section{WEB SERVICE BASED ARCHITECTURE}

The required distributed approach is implemented by a service oriented architecture (SOA). It is the basis for web services, where the functionality is described in distributed pieces of software accessible over a network, e.g. the Internet. In order web services exist with in a SOA, a common mechanism to describe, discover and invoke these distributed software methods is required.

Three basic roles and their relations describe the SOA, as pointed out in Figure 4a: Service registry, service consumer and service provider, including the web service description, explained in more detail in [11].

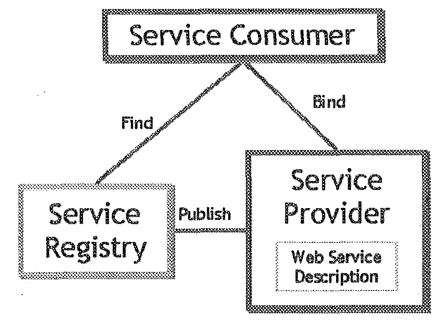

a)

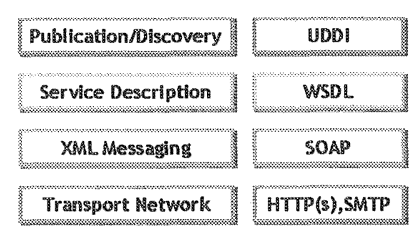

b)

Figure 4-a) SOA and b) Web service stack

The interoperable web service stack describes the functionality behind the SOA. The web service stack employs platform and vendor independent standards like Universal Description, Discovery and Integration (UDDI), Web Service Definition Language (WSDL), Simple Object Access Protocol (SOAP) and HTTP/SMTP etc., as shown in Figure 4b. In this case, SOAP is the XML-based protocol for invoking web services. WSDL is a standard for describing web services. UDDI is the mechanism for publishing and discovering web services by providing a kind of registry making use of WSDL.

These software technologies are used to enable distributed pieces of software to communicate in a predefined manner to each other. The details of these technologies are well described in $[12,13,14]$. For the development described in this paper, these technologies are used to enable XML-based cooperative engineering based on an infrastructure, integrating vendors within a plant production project. 
The company driven requirements for cooperative work, e.g. transparency of really transferred data, security, and (a -) synchronous communication are met by the use of XML-based standards. SOAP supports, based on remote procedure calls (RPC) and messaging, synchronous as well as asynchronous transfer protocols. The latest incarnation of Apache SOAP, called Apache eXtensible Interaction System (AXIS) [15], enables an integration of the required flexible security. For that and other reasons, AXIS provides an appropriate basis for the implementation of the later on described example.

To demonstrate the cooperative engineering research and the architectural investigation in conjunction with security enabling encryption and signing methods a practical application in the fields of plant production was chosen as a test case.

In the following section based on a detailed description of the structure for the cooperative engineering supporting software in the fields of plant production the test case is performed with a popular planning software and a famous robot simulation tool.

\section{SECURE COOPERATIVE ENGINEERING}

As mentioned in the state of the art concerning accomplishments the establishment of knowledge protection is important as well as data transfer security to enable cooperative engineering required by digital factory approaches as described in [16]. This results in the described view technology and requires the use of encryption and decryption methods as well as digital signature mechanisms. The required security methods enable authentication, authorisation, data integrity and privacy and non-repudiation concerning every transmitted information. The authentication, authorisation and partly the non-repudiation are considered inside the developed web services for the cooperative engineering software prototype. The data integrity and privacy deals mainly with encryption and decryption. These methods are implemented and integrated into the web service architecture. As a special function they are previously called to every deployed web service, if the call is performed from outside of a determined boundary. In addition to the security issues, the research on cooperative work in the fields of plant production as described in $[16,17]$ and the use of a XML-based SOA result in a reliable trustworthy software prototype for cooperative engineering. Based on the basic web service structure and the architectural model including the security methods, the example environment is described.

\section{Architecture with integrated security}

Each of the developed web services is independent on the used security method. The use of the AXIS based web service architecture, described in Figure 5, resulted in an investigation of security enabling methods, for an appropriate encryption and decryption of the transferred SOAP messages.

Two types of encryption are available: symmetric and asymmetric. The symmetric encryption is performed based on a common key at each partner. For that reason, the key has to be known by the sender and the receiver of an symmetrically 
encrypted message. The symmetric key is built at one partner. The main problem occurring with the use of symmetric encryption the secure distribution of the symmetric key to the relevant partners. To solve this key exchange problem a asymmetric encryption is an appropriate method. To establish a secure communication between the partners using an asymmetric method each partner needs a pair of public and private key. The public key is published to the partners. With the public key of the receiving partner the sensitive information is encrypted. Only that partner with the related private key is able to decrypt the message. The symmetric method is a lot faster than the asymmetric procedure as explained in [18].

Against this background an appropriate combination of both methods is required for a secure transfer of SOAP messages. The symmetric encryption is applied to the information containing comparatively huge body of the SOAP message. The related symmetric key is included into the header of the same message and encrypted by an asymmetric encryption method. The management of the related keys for the asymmetric method is performed by an external public key infrastructure (PKI). To ensure a reliable application of security, each invocation of a web service has to result into a previously called method for encryption or decryption. For that reason the SOAP message has to be processed in front of the original call of each web service.

This procedure is implemented into AXIS by using a special architectural property, the handler chains, presented in Figure 5. The security enabling methods based on XML-Encryption[19] and XML-Signature[20] are integrated into a handler. The decryption handler is responsible for processing received SOAP messages. The encryption handler processes the "to send" messages. After decryption a signature checking handler is called. This ensures that all the security related requirements are met.

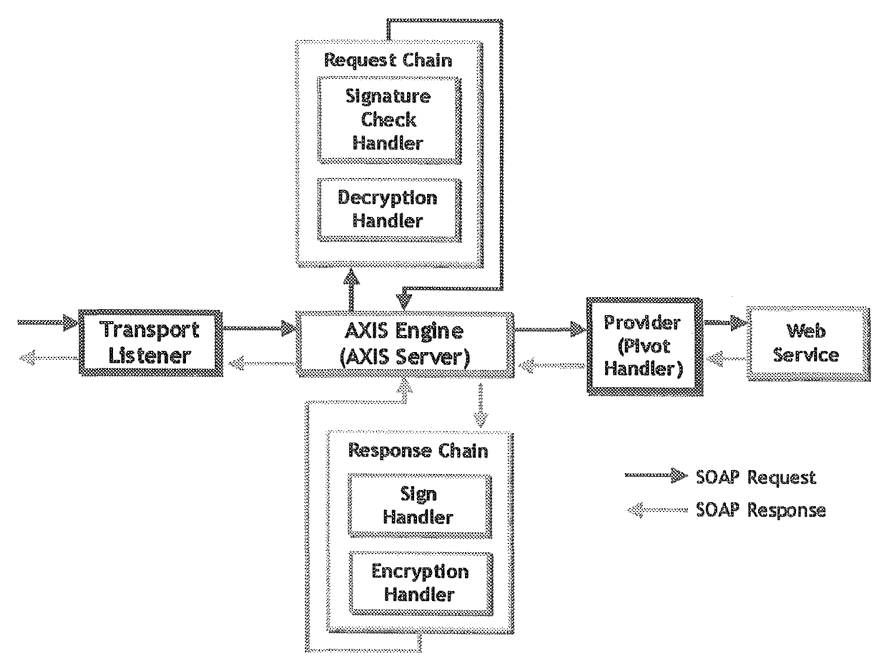

Figure 5 - Simplified description of the extended AXIS architecture 
For demonstration purposes the advanced encryption standard algorithm (AES) [21] was used as an example symmetric method with a key length of $128 \mathrm{k}$ and $256 \mathrm{k}$. For implementation of the asymmetric procedure the RSA algorithm [21] was chosen with a key length of $1024 \mathrm{k}$. For the signature the digital signature algorithm (DSA) [21] is used. This enables a sufficient security for the information exchange within a plant production environment

The main advantage of this kind of integrating security methods is the flexibility in location of the web services used for cooperative engineering. This flexible location of the web services and the flexible location of the models demonstrate that distributed secure cooperative engineering in plant production is possible.

\section{Web Service Structure}

The decision to use XML based model descriptions as a basis for the model exchange, entailed the set up of an appropriate XML schema, described in [22], for the often proprietary data formats of all involved software tools. This prerequisites an XML interface at each considered software tool. In the case of the example described in this paper, the used planning software does already include an XMLbased interface that can be used for the purpose of integration.

The mentioned robot simulation application missed an appropriate XML interface. For that reason a detailed XML based interface for the robot simulation was developed. Based on these XML interfaces the developed web services are able to access the models residing in the respective legacy software tools. For semantically conversions the view mechanism transforms the XML-based model into the required structure. The related transformation policies and their application are stored together with the related class of each XML-based model.

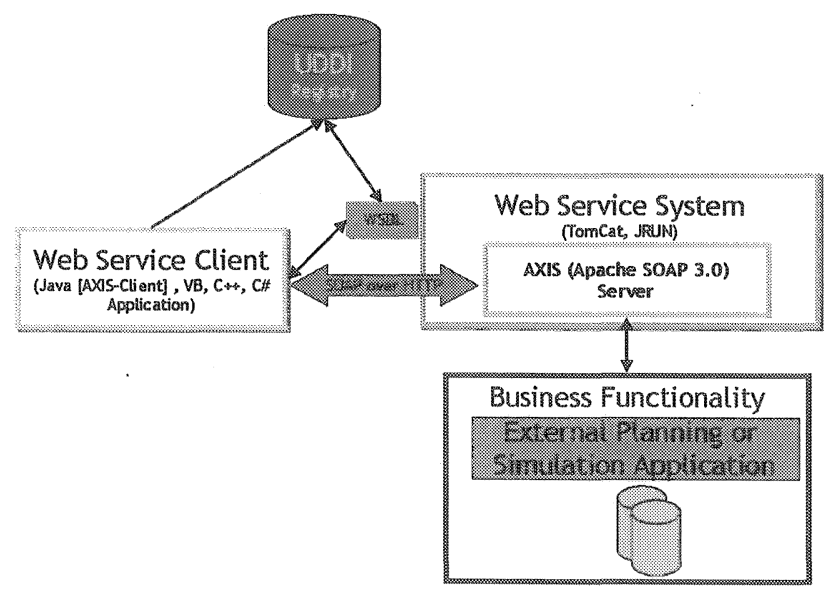

Figure 6 - General web service structure

Based on a general web service structure, shown in Figure 6, further research and a detailed requirements analysis resulted into a certain web service structure for plant production purposes, visualised in Figure 7. 


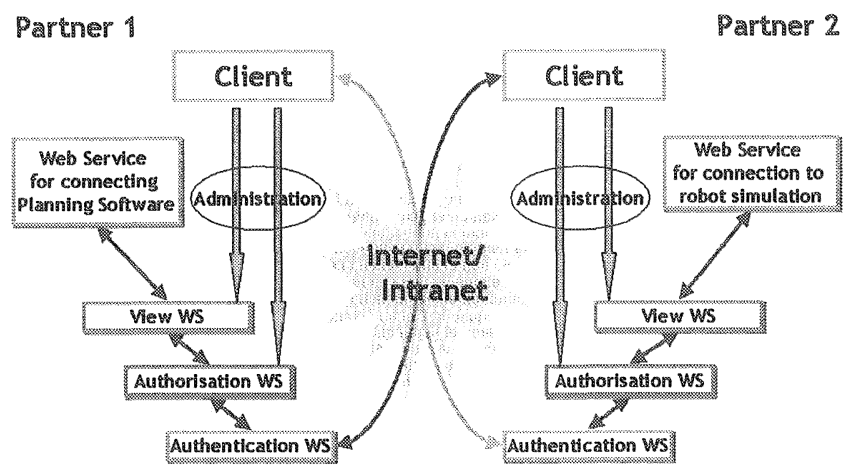

Figure 7 - Cooperative work web service structure

AXIS is used for implementation of the example environment, as mentioned. For that reason, at each cooperation partner an AXIS based or a SOAP compatible client including a model view browser exists. In addition, an AXIS server reacting on incoming SOAP messages is installed. In the example, later described, the cooperative work enhancing system is based on AXIS only, because of the availability as open source software.

The different tasks required for authentication, authorisation, view technology and the integration of an external application are separated into several web services running at each partner. With the architecture shown in Figure 7, an application of a peer-to-peer (P2P) structure is facilitated with a modern software concept. To keep the overview, the AXIS internal architectural extensions for enabling a secure SOAP-based communication, the respective handlers, are not mentioned in Figure 7. Every communication is performed based on XML-based encryption and decryption based on AXIS handlers as described above.

\section{Example Application Environment}

A SOA is an abstract model, but several company driven definitions, standardised by the W3C, enable concrete software developments, as shown by the research and development results presented in this paper, particularly in Figure 5 and Figure 7.

By deployment of a SOA approach, the web service based P2P application prototype consists of a client application, a UDDI-based registry for cooperation partners including their web services and the web services themselves, enabling authentication, authorisation, views and legacy software integration. The concept allows the distributed location of the software functionality and models as well as an encrypted communication. In the following, an example workflow is presented, describing the procedure for model exchange in a real life cooperation.

A user in a company is requesting a robot model. The model resides at a cooperation partner. A typical today's situation is that this cooperation partner asks for the model via Email or Fax and often gets the results in form of paper ware. This is not very efficient, because of the expensive integration process into the existing 
local software system. On this account, the described software system was developed that allows a flexible and digital way of exchanging models.

The cooperative engineering software enables the user to request the robot model residing at another partner. This request is split up into two steps. In a first step, the registry is called to obtain the location of the requested model and the responsible partner. In a second step, the located web service at the respective partner, providing the requested robot model, is invoked via a SOAP Message. Within this invocation the user data is sent to determine the users identity and to authorise the users affiliated role dependent on the company and the related project. After receiving the request, the server authenticates the user, authorises the role in respect to the mentioned dependencies and defines the view to the model that afterwards will be applied by the view building web service. This view builder requests the full model using the developed XML-based interface from the robot simulation tool XML-based interface.

In a next step, it builds the view as previously administrated and replies the resulting XML-based model to the requesting cooperation partner. Within this scenario every request and each reply is encrypted and signed to provide the required non-repudiation, privacy and data integrity to enable reliable cooperative engineering. At the client side the model can be viewed and inserted into the integrated planning software.

All cooperation partners are able to work on models with their legacy application, exchanging those models via the described software system. On the one hand this decreases the costs superseding software tools enabling cooperative engineering and on the other hand companies with an existing, good working software architecture are not willing to exchange this because of cooperation purposes only.

\section{CONCLUSIONS}

The main goal to enhance cooperative engineering in plant production processes was achieved by the use of a service-oriented architecture (SOA) in conjunction with security investigations, research in cooperative engineering and an industrial driven requirements analysis. In the future an Assembly of the derived research and development results with an enhanced workflow and model management into test case environments in an industrial real-time setting will be performed. As testing the developed methods together with model management and an appropriate workflow management, we cannot yet draw final conclusions. For this reason, this section will describe the advantages derived and expected.

Defining and modelling the considered activities and then ensuring the needed interactions between these activities has two main advantages. Obviously the needed transfer of information has to be possible. Perhaps, less clear is that no effort is wasted on interactions that are not required. The approach of reducing the communication efforts and an increase in automated inter-company cooperative engineering between the activities involved into a cooperation enables a higher reliability in the whole plant production process. 
The use of XML results in an easy extensible software architecture. Company dependent clients, integrated into the in-house legacy architecture are easy to build. The software described in this paper can be flexibly adopted to company philosophies and structures because of the separation into several distributed modules, the web services. Future enhancements are able to integrate easily at any position inside the developed software system for plant production purposes.

The results were demonstrated in an example scenario, integrating different engineering applications. An everywhere around the world usability, because of a web service based system ensures the required flexibility for wide spread locations of a company or of the project partners. The software is not superseding existing collaboration tools. It is one of the future tasks to integrate facilities for direct contact between the cooperation partners.

With the described approach, the first steps towards cooperative engineering of a whole production facility are accomplished. By now it can be assumed, that the increase of efficiency in engineering production facilities together with partners, will result in positive business effects like decrease in terms of cost and time-to-market. The time for start up the real production facility can be extremely reduced, because the main work, the adaptation of software and configuration of mechanical parameters, is mainly done in front of the start up by teams within the virtual world.

\section{ACKNOWLEDGEMENTS}

Some of the developments and research described in this paper is being performed within the VIDOP project: "Vendor Integrated Decentralised Optimisation of Production facilities" (VIDOP, G1RD-CT2000-00301) [1]. This is a shared cost project partially supported and funded by the European Commission's Competitive and Sustainable Growth Programme within the fifth Framework.

\section{REFERENCES}

[1] VIDOP: Vendor Integrated Decentralised Optimisation of Production facilities, Project description: http: //vidop.ipr.uka.de, 2001

[2] J.P. Harrison, B. Christensen, J. Bianco, M. Gulli, Virtual Collaborative Simulation Environment for Integrated Product and Process Development, High Performance Distributed Computing, Proceedings of $5^{\text {th }}$ IEEE International Symposium, Deneb Robotics Inc., Auburn Hills, MI, pp $19-22,6-9$ Aug. 1996

[3] J. Barry, M. Aparicio, T. Durniak, P. Herman, J. Karuturi, C. Woods, C. Gilman, H. Lam, R. Ramnath, NIIIP-SMART, An Investigation of Distributed Object Approaches to Support MES Development and Deployment in a Virtual Enterprise, EDOC'98 - The $2^{\text {nd }}$ International Enterprise Distributed Object Computing Workshop, La Jolla, California, USA, November 3-5, 1998

[4] A.V. Smirnov, C. Chandra, Ontology-Based Knowledge Management for Co-operative Supply Chain Configuration, AAAI Spring Symposium, Technical Report SS-00-03 "Bringing Knowledge to Business Processes", ISBN 1-57735-109-6, pp 85 - 92, 2000

[5] IEEE 1516 DRAFT Standard, IEEE SA., Denise Pribula IEEE SA, d.pribula@ieee.org 
[6] J. Woerner, T. Laengle, H. Woern, Corporate Planning and Simulation of Plant Production Facilities in the Virtual World, Proceedings of the $18^{\text {th }}$ International Conference on CAD/CAM, Robotics and Factories of the Future - CARs\&FOF, Porto, Portugal, pp. $109-1162002$.

[7] H. Salmi, V.K. Tuunainen, Competitive Co-operation Facilitated by Electronic Comerce, Proceedings of the IRIS 23 Conference, Laboratorium for Interaction Technology, University of Trollhättan, Uddevalla, Sweden, 2000

[8] P. Leitão, J. Barata, L. M. Camarinha-Matosand, R. Boissier, Trends in Agile and Cooperative Manufacturing, Proceedings of Low Cost Automation Symposium, Berlin, Germany, 8-9 October, 2001.

[9] P. Leitão, F. Restivo, An Agile and Cooperative Architecture for Distributed Manufacturing Systems, Proceedings of the IASTED International Conference Robotics and Manufacturing, Cancun, Mexico, pp 188 - 193, 21-24 May, 2001

[10] K. R. Caskey, K. Rouibah, Integrating and Managing Vendor Supplied Models of Production Facilities, Proceedings of the $18^{\text {th }}$ International Conference on CAD/CAM, Robotics and Factories of the Future-CARs\&FOF, Porto, Portugal, pp. 185 - 192, 2002.

[11] K. R. Caskey, K. Rouibah, H.-J. Pels, Managing Vendor Supplied Models of Production Facilities, European Simulation Interoperability Workshop, University of Westminster, London, England, 25-27 June, 2001

[12] P. Cauldwell, R. Chawla, V. Chopra, C. Dix, G. Damschen, T. Hong, F. Norton, U. Ogbuji, G. Olander, M. Richman, K. Saunders, Z. Zaev, Professional XML Web Services, Wrox Press, 2001

[13] M. Hendricks, B. Galbraith, R. Irani, J. Milbery, T. Modi, A. Tost, A. Toussaint, S. J. Basha, S. Cable, Professional Java Web Services, Wrox Press, 2002

[14] S. Brodhead, A. Cioroianu, J. Hart, E. Jung, D. Writz, Professional Java XMLProgrammers Reference, Wrox Press, 2001

[15] Apache Project: Apache eXtensible Interaction System - AXIS, Documentation for xmlaxis-beta3 release, http: //xml.apache.org/axis/, 2002

[16] H. Woern, D. Frey, J. Keitel, Digital Factory - Planning and Running Enterprises of the Future, Proceedings of the 26th Annual Conference of the IEEE Electronics Society IECON-2000, pp. 1286 - 1291, IEEE Press, 2000

[17] J. Woerner, T. Laengle, Co-operative Work in Plant Production Processes, VIDOP Workshop Porto, 26-30 September, 2001

[18] Nash, W. Duane, C. Joseph, D. Brink, PKI Implementing and Managing E-Security, RSA Press, ISBN: 0-07-213123-3, Osborne/McGraw-Hill, 2001

[19] World Wide Web Consortium (W3C), XML-Encryption Working group, http: //www.w3.org/Encryption/2001/, 11 June, 2002

[20] Internet Engineering Task Force (IETF), World Wide Web Consortium (W3C), XMLSignature WG, available at http: //www.w3.org/Signature/, 11 July, 2002

[21] B. Schneier, Secrets and Lies - Digital Security in a Networked World, John Wiley \& Sons, $1^{\text {st }}$ Edition, 2000

[22] World Wide Web Consortium (W3C), XML Schema Ver. 1.1 Recommendation, http: //www.w3.org/XML/Schema, 2001. 